\title{
Unequal Error Protection Irregular Over-Complete Mapping for Wavelet Coded Wireless Video Telephony Using Iterative Source and Channel Decoding
}

\author{
A. Q. Pham, L. L. Yang, and L. Hanzo \\ School of ECS, University of Southampton, SO17 1BJ, UK \\ Tel: +44-23-8059 3125, Fax: +44-23-8059 4508 \\ Email:\{apq03r,lly,lh\}@ecs.soton.ac.uk, http://www-mobile.ecs.soton.ac.uk
}

\begin{abstract}
Since the different bits of the compressed video sequence produced by the BBC's wavelet-based video codec exhibit different error sensitivity, efficient Unequal Error Protection (UEP) schemes have to be used to ensure that the perceptually more important bits benefit from more powerful protection. Furthermore, in the context of turbo detection the over-complete mapping aided soft videobit based source decoder should also match the characteristics of the channel codes for the sake of attaining a good iterative decoding performance. In this paper, we address this design dilemma by proposing a novel Irregular Over-complete Mapping (IrOM) scheme constituted by an appropriately weighted different-rate over-complete mapping. Our turbo detection scheme has attractive iterative decoding convergence properties, while maintaining unequal error protection capabilities matched to the requirements of the wavelet encoded video sequence. An EXIT chart based design guideline is presented and used in the context of protecting the different-sensitivity video bits of the $\mathrm{BBC}$ wavelet-based video codec. As a benefit, the unequal error protection scheme using rate $2 / 3$ irregular over-complete mapping exhibits a transmit power advantage of about $0.7 \mathrm{~dB}$ at an error probability of $0.016 \%$ over the equal-protection benchmarker scheme employing rate $2 / 3$ over-complete mapping, when communicating over the uncorrelated Rayleigh-fading channel. This leads to an approximately $1 \mathrm{~dB}$ signal-to-noise ratio gain, when considering the video PSNR performance of our UEP iterative source-channel decoding scheme using an overcomplete mapping having the same rate and $I=6$ iterations.
\end{abstract}

Index Terms-generalized low-density parity-check codes, iterative source-channel decoding, extrinsic information transfer charts, over-complete mapping, irregular

The financial support of the EPSRC, Swindon UK and the EU, Brussels is gratefully acknowledged. over-complete mapping.

\section{INTRODUCTION}

The intentionally imposed or inherent unintentional residual redundancy found in source encoded multimedia sequences can be exploited for supporting joint softbit source decoding and channel decoding, which has the potential of improving both the error correcting capability as well as the subjective audio or video quality of communication systems. This has been successfully exploited in the over-complete mapping aided iterative source-channel decoding scheme of [1], which exploits both the residual redundancy inherent in the video encoded sequence generated by the BBC's open-source Dirac codec [2] as well as the intentional redundancy imposed by over-complete mapping (OM) for the sake of mitigating the effects of transmission errors.

However, the Dirac video-encoded sequence exhibits different bit error sensitivities for different bit positions. When aiming for low-bitrate operation, the bitrate contribution of the fixed-length header information becomes quite dominant, for example about $30 \%$ of the total frame size of 2000 bits. Naturally, this is the most errorsensitive segment of the encoded video frame, since no useful information can be decoded in its absence. Additionally, the employment of Context-based Adaptive Binary Arithmetic Coding (CABAC) [3] renders the compressed video sequence sensitive to channel errors, since both the CABAC technique as well as the predictive coding inflict error propagation upon both the future decoded subbands' coefficients of the current video frame and on the future video frames. Hence, if the transceiver has the knowledge of the source bit error 
sensitivities, Unequal Error Protection (UEP) typically performs better than Equal Error Protection (EEP) by applying a lower channel coding rate to the more sensitive portions of the data sequence, and a higher channel coding rate to the less sensitive portions, while keeping the overall channel coding rate the same as that of the EEP scheme.

As a novel contribution of this paper, we design a serially concatenated turbo transceiver using Irregular Over-complete Mapping (IrOM) for Dirac-encoded video telephony. Rather than being decoded separately, the constituent over-complete mapping schemes of the IrOM are decoded jointly and iteratively by exchanging extrinsic information with the rate $2 / 3$ Generalized LowDensity Parity-Check (GLDPC) code of [4] used as our inner code. The IrOM is optimized to match the characteristics of both the Dirac codec and those of the rate 2/3 GLDPC code, so that UEP scheme maximizes the achievable iteration gain and the resultant system performance.

The rest of the paper is organized as follows. In Section II, the schematic of an iterative detection aided unequal error protection Dirac videophone scheme using IrOM is presented. In Section III, we investigate the design of IrOM in the context of Dirac-encoded video transmission and analyse its performance using EXIT charts. Our overall system performance results are provided in Section IV to demonstrate that the UEP scheme outperforms the EEP scheme in terms of the achievable Peak Signal-to-Noise Ratio (PSNR). Finally, our conclusions are provided in Section V.

\section{System Model}

The block diagram of the iterative detection aided unequal error protection Dirac videophone scheme using IrOM is shown in Fig. 1. At the transmitter, the Dirac wavelet video codec described in [2] is employed for compressing the video frames. The video-encoded binary sequence $x_{k}, \quad i=1,2, \ldots, N$ is mapped to the binary sequence $z_{i}, \quad i=1,2, \ldots, P$ using the irregular overcomplete mapping to be designed in Section III. The resultant sequence $z_{i}$ is then interleaved using the bitinterleaver $\Pi$ of Fig. 1 and subsequently the interleaver's output sequence $\bar{z}_{i}, \quad i=1,2, \ldots, P$ is encoded by the Generalized Low-Density Parity-Check (GLDPC) Code of [4], before its transmission over an uncorrelated Rayleigh fading channel.

At the receiver, the soft-decision based reliability information $\hat{y}_{j}, \quad j=1,2, \ldots, M$ is evaluated in an iterative 'turbo-detection' fashion, which exchanges extrinsic

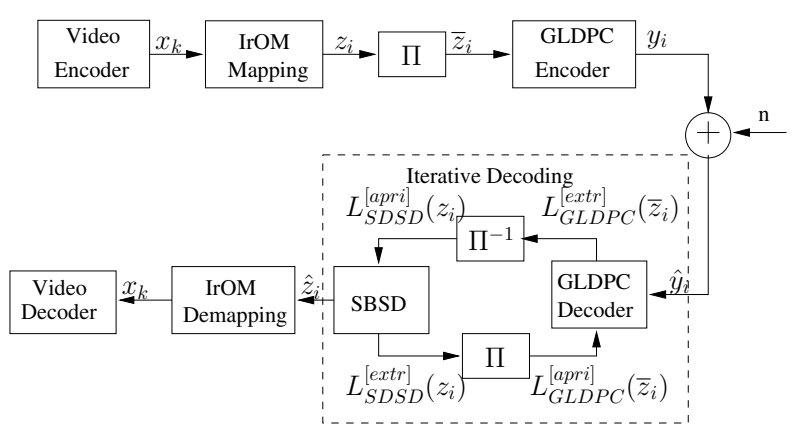

Fig. 1. System model of an iterative detection aided unequal error protection Dirac videophone scheme using Irregular Over-complete Mapping (IrOM).

information between the GLDPC decoder and the softbitsource decoder of [1]. This reliability information can be evaluated either in terms of bit probabilities or as their log-likelihood ratios (LLRs). At the last iteration softbit-estimation is carried out, the resultant bit stream is demapped and then fed into the Dirac video decoder for the sake of reconstructing the video sequence.

\section{Design of IRREgular OVER-COMPlete MAPPING}

In this section, we exploit the knowledge of the Dirac video bit error sensitivity of [2] for designing an iterative detection aided unequal error protection Dirac videophone scheme. More specifically, at the transmitter side we invoke data partitioning, which partitions the Dirac video encoded sequence into classes of bits having different error-sensitivities and then apply a lower-rate over-complete mapping for protecting the more sensitive classes, and a higher-rate over-complete mapping for the less sensitive classes, while keeping the overall rate of the IrOM arrangement the same as that of the EEP scheme. More explicitly, an IrOM arrangement may be constructed by employing a family of over-complete mapping schemes having rates of $\left[r_{1}, r_{2}, \ldots, r_{P}\right]$. Let $L$ denote the total number of mapped bits generated by the IrOM from a group of $K$ input information bits. Each constituent mapping maps a fraction of $\alpha_{k} \cdot r_{k} \cdot L, k=$ $1, \ldots, P$ information bits and generates $\alpha_{k} \cdot L$ mapped bits. Given the target code rate of $R \in[0,1]$, the weighting coefficient $\alpha_{k}$ has to satisfy:

$$
\sum_{k=1}^{P} \alpha_{k}=1, \quad \sum_{k=1}^{P} \alpha_{k} \cdot r_{k}=R, \quad \text { and } \alpha_{k} \in[0,1] \forall k .
$$

Note that rather than being decoded separately, the mapped symbols of constituent over-complete mappings of the IrOM are decoded jointly and iteratively by exchanging extrinsic information with the inner GLDPC 
code. Furthermore, the characteristics of the Dirac codec and those of the GLDPC code are exploited, in order to optimize the IrOM scheme, so that UEP is achieved while maximizing the iteration gain of the system.

\section{A. Source-Coding Assisted Design of IRCCs}

In this section, we design the UEP scheme by appropriately matching the IrOM to the Dirac video sequence. In our work, we first partition the unequalsensitivity video-encoded sequence into a number of protection classes and then invoke IrOM in order to protect them differently. Based on the bit error sensitivity investigations of [2], our partitioning algorithm splits the Dirac video frame into three classes, namely the Most Significance Bits (MSB), Very Significance Bits (VSB) and the Least Significance Bits (LSB). The specific class, where a certain bit is assigned to is dynamically decided as follows:

1) The video header bits, including the frame header, motion vector header and all 30-subbands' headers, are MSBs. An error corrupting the MSBs will result in the decoder's loss of synchronization with the encoder and hence results in catastrophic decoding failure of the subsequent process.

2) All bits of the motion vectors are assigned to the LSB class, if the number of motion vector bits is less than 500. This is, because it was documented in [2] that despite their inter-frame propagation an error in any of them will inflict a relatively low distortion in the decoded frame, which results in a PSNR degradation of less than $8 \mathrm{~dB}$.

3) All bits of the subband video bits are also deemed to be LSBs, when the number of subband bits is less than 500. The video PSNR degradation imposed by the corruption of any of them is typically confined to the range below $8 \mathrm{~dB}$.

4) By contrast, the motion vector and subband parameter encoding bits are split into two different classes, if they generate more than 500 bits. More specifically, the least sensitive 500 bits are considered as LSBs, while the remaining bits will be deemed to be VSBs.

Although the three protection classes are interleaved during the encoding and decoding process, their classifier information (whether they are MSBs, VSBs or LSBs) is recovered by the decoder based on the side-information provided by the MSBs.

Furthermore, a simple packetization scheme concatenates these classes in order to form fixed-length packets to transmit. It is worth noting that due to the various sizes of the protection classes and owing to the employment of fixed-length component OMs by the IrOM scheme, the concatenation of these classes may lead to using different mapping rates within a specific protection class.

TABLE I

THE AVERAGE RATIO OF THE THREE CLASSES IN THE DiRAC VIDEO SEQUENCE.

\begin{tabular}{|l|c|c|c|}
\hline \multirow{2}{*}{} & \multicolumn{3}{|c|}{ The relative frequency of occurrence of } \\
\cline { 2 - 4 } & MSBs & VSBs & LSBs \\
\hline Probability (\%) & 30.41 & 19.77 & 49.82 \\
\hline
\end{tabular}

An interesting question arising for the design of the IrOM is how to decide the ratio of the bits assigned to each component $\mathrm{OM}$ of the IrOM. The basic criterion is to aim for keeping the same ratio as in the original Dirac video encoded sequence. Unfortunately, this ratio is time-varying. Table I shows the ratio of the differentsensitivity bits computed for the Dirac-encoded QCIF "Miss America" video sequence scanned at 30 frame/s. The ratio of these bits is depicted in Figure 2. We can see from Figure 2 that although the ratio varies from frame to frame, their range is limited. Hence, we opted for choosing a ratio that guarantees that the MSB class is protected by the lowest-rate over-complete mapping, while the VSB and LSB class should be protected by a higher-rate over-complete mapping.

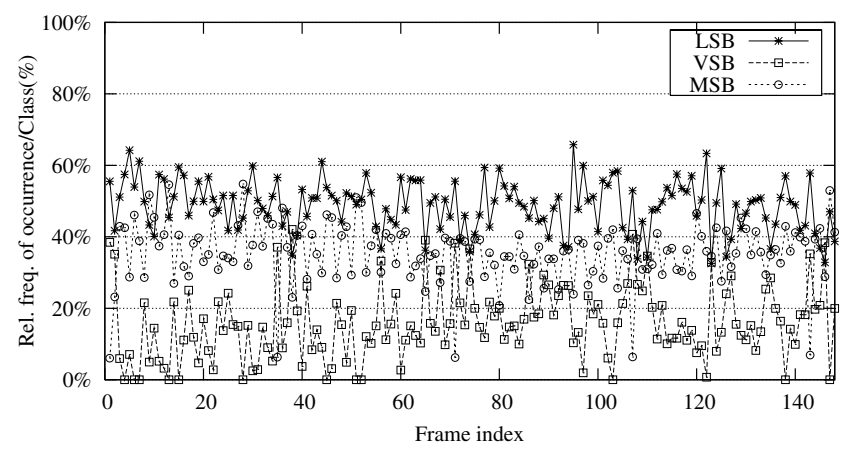

Fig. 2. The relative frequency of occurrence for the MSB, VSB, LSB Dirac video bits computed from the "Miss America" QCIF video sequence encoded at 30 frame/s.

Another factor in deciding this ratio is the fairness of our comparisons. More specifically, in order to compare the performance of the EEP and UEP schemes, we have to let the rate of IrOM and that of the single overcomplete mapping arrangement to be the same. More explicitly, the EEP and UEP schemes are chosen as follows

1) For the EEP scheme, a rate-6/9 over-complete 
mapping was employed as the outer code, which provides equal protection for the video bits.

2) For the UEP scheme, we employ an IrOM that consists of a family of component OMs having rates of $\left[\frac{6}{7}, \frac{6}{10}, \frac{6}{11}\right]$, which encode $[840,510,660]$ video bits, respectively. This results in the IrOM having a coding rate of $2 / 3$ for $K=2010$ bits of video bits per transmitted packet.

\section{B. Convergence Analysis Using EXIT Charts}

Let us first investigate the EXIT characteristics [5] of the various over-complete mapping components of the IrOM scheme. In all cases, the EXIT curves seen in Fig.3 were generated using uncorrelated Gaussian distributed $a$ priori LLRs with the assumption that the 6-bit source symbols ${ }^{1}$ have uniform probability distribution. All EXIT transfer functions were obtained by using the histogram-based LLR measurements of [5].

For the IrOM decoder (outer decoder), we denote the mutual information between the a priori input $A$ and the mapped bits $C$ as $I_{A}^{o}=I(C ; A)$, while the mutual information between the extrinsic output $E$ and the mapped bits $C$ is denoted as $I_{E}^{o}=I(C ; E)$. Then the transfer function of outer decoder can be defined as:

$$
I_{E}^{o}=T_{o}\left(I_{A}^{o}\right)
$$

which maps the input variable $I_{A}^{o}$ to the output variable $I_{E}^{o}$. Similarly, for the GLDPC decoder (inner decoder), we denote the mutual information between the a priori input $A$ and the transmitted information bits $X$ as $I_{A}^{i}=$ $I(X ; A)$. Furthermore, we denote the mutual information between the extrinsic output $E$ and the transmitted information bits $X$ as $I_{E}^{i}=I(X ; E)$. Note that the extrinsic output of the inner code also depends on the channel SNR or $E_{b} / N_{0}$. Hence the transfer function of the inner code is defined as:

$$
I_{E}^{i}=T_{i}\left(I_{A}^{i}, E_{b} / N_{0}\right)
$$

We also denote the EXIT transfer function of the $k^{\text {th }}$ component softbit-source decoder $k=1,2,3$ of the amalgamated IrOM decoder as $I_{E}^{o, k}=T_{o, k}\left(I_{A}^{o, k}\right)$. The inverted transfer functions $I_{A}^{o, k}=T_{o, k}^{-1}\left(I_{E}^{o, k}\right)$ of the IrOM's component softbit-source decoders and that of the IrOM decoder, namely $I_{A}^{o}=T_{o}^{-1}\left(I_{E}^{o}\right)$ are again depicted in Fig. 3.

\footnotetext{
${ }^{1}$ All bits of the MSB, VSB and LSB classes were assigned to 6-bit source-symbols, which were then protected by the $[6 / 7,6 / 10,6 / 11]$-rate OMs
}

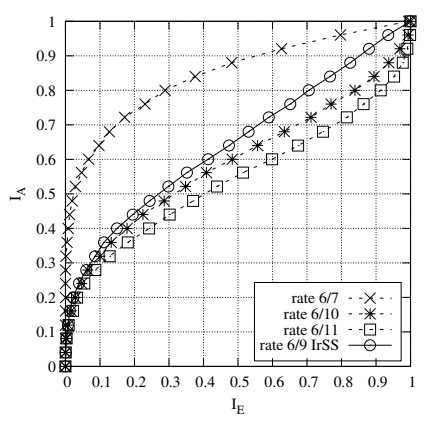

Fig. 3. The EXIT transfer function of the rate-2/3 IrOM and that of its components

Note that the EXIT transfer function of the IrOM decoder can also be obtained as the weighted superposition of the $P=3$ component softbit-source decoders' transfer functions as follows

$$
T_{o}\left(I_{A}^{o}\right)=\sum_{k=1}^{P} \alpha_{k} \cdot T_{o, k}\left(I_{A}^{o}\right),
$$

where $\alpha_{k}$ is the weighting coefficient mentioned above. The EXIT charts [5] of the UEP scheme using IrOM as well as the EEP scheme using rate-6/9 over-complete mapping are shown in Fig. 4.

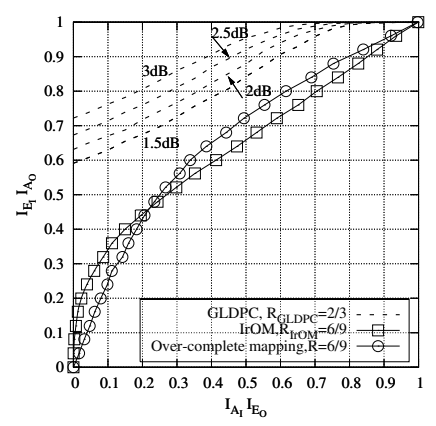

Fig. 4. The EXIT transfer functions of the rate-6/9 over-complete mapping aided outer decoder and the designed IrOM decoder, as well as that of the inner decoder at $E_{b} / N_{0}=1.5,2.0,2.5$ and $3 \mathrm{~dB}$.

As seen from the EXIT chart, there is a wider EXIT chart tunnel between the inner decoder's curve and the outer decoder's curve for the proposed UEP scheme in comparison to the EEP scheme using the rate-6/9 over-complete mapping. Hence, given a limited number of iterations, we would predict that the UEP system using the IrOM may be expected to perform better than the EEP scheme employing the rate-6/9 over-complete mapping in the range of $E_{b} / N_{0}=1.5 \ldots 3 \mathrm{~dB}$. 


\section{EXPERIMENTAL RESULTS}

In this section, the attainable performance of the proposed UEP scheme of Fig. 1 is investigated for transmission over an uncorrelated Rayleigh fading channel. The various system parameters are summarized in Table II.

TABLE II

THE SIMULATION PARAMETERS

\begin{tabular}{|l|r|r|}
\hline Parameters & EEP scheme & UEP scheme \\
\hline Overall code-rate & 0.45 & 0.45 \\
Video codec & Dirac & Dirac \\
Mapping rate & $6 / 9$ & $6 / 9$ \\
Bit-Interleaver $\Pi_{1}$ & 3040 bits & 3040 bits \\
GLDPC code-rate & $2 / 3$ & $2 / 3$ \\
Constituent codes & BCH(12,10,1) & BCH(12,10,1) \\
Modulation & BPSK & BPSK \\
\hline
\end{tabular}

The associated BER performances are shown in Fig. 5 for an overall code-rate of 0.45 . More specifically, the proposed UEP system outperformed the EEP system by about $0.7 d B$ for $I=6$ iterations. The video PSNR

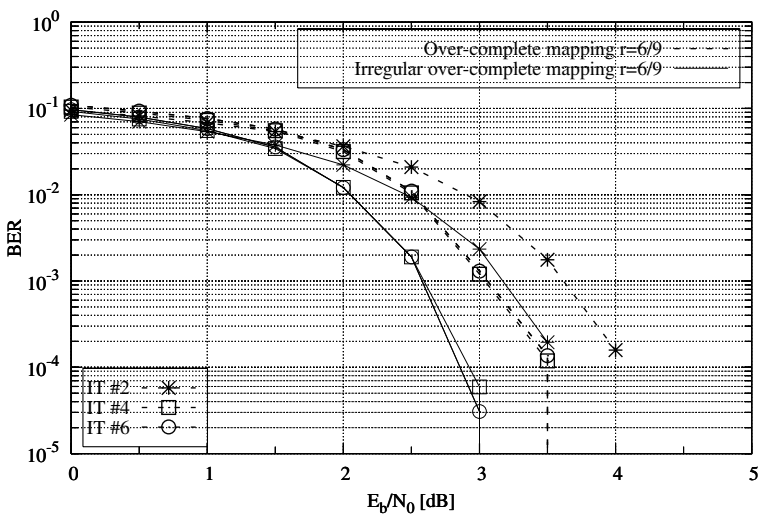

Fig. 5. BER performance of the EEP system and the UEP system of Table II designed for transmission over the uncorrelated Rayleigh fading channel.

performances of the UEP scheme and EEP scheme summarized in Table II are depicted in Fig. 6. Observe from Figs. 5 and 6 that our UEP system attains a nearunimpaired video reconstruction quality for a channel $E_{b} / N_{0}$ value of $2.75 \mathrm{~dB}$, although at this point the overall BER is about $1.6 \cdot 10^{-4}$. Furthermore, at the same channel $E_{b} / N_{0}$ value of $3.25 \mathrm{~dB}$ the video reconstruction quality of the EEP system is significantly lower than that of the UEP system, although at this point the overall BER of the EEP system is about $5 \cdot 10^{-4}$.

\section{Conclusions}

Novel irregular over-complete source-encoding mapping schemes were proposed for video transmission over

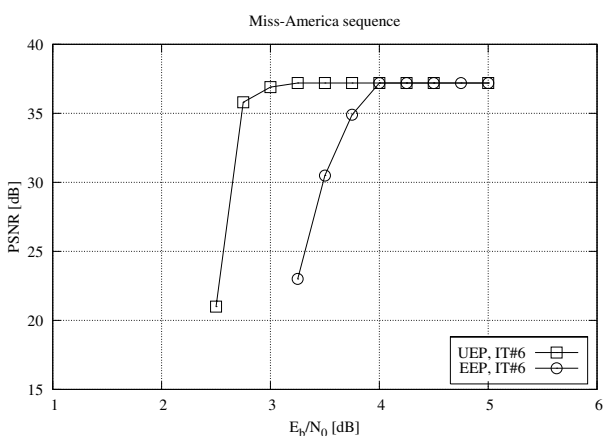

Fig. 6. Comparison of the achievable video PSNR using the systems characterized in Table II.

the uncorrelated Rayleigh fading channel. More specifically, we exploited the knowledge of the Dirac codec's video bit error sensitivity [2] for designing an irregular over-complete mapping scheme, which was combined with partitioning the Dirac video encoded sequence into 3-classes of bits having different sensitivities. We applied a lower rate over-complete mapping for protecting the more sensitive classes, and a higher rate over-complete mapping arrangement for the LSB class, while keeping the overall rate of IrOM the same as that of the EEP scheme. It was found that the error correcting capability of the resultant UEP scheme using IrOM has been improved. Our experimental results seen in Fig. 5 and 6 demonstrate that at the same overall system code-rate of 0.45 , the UEP scheme designed with the aid of our irregular over-complete mapping outperforms the EEP scheme using over-complete mapping by about $1 \mathrm{~dB}$.

\section{REFERENCES}

[1] A. Q. Pham, L. Hanzo, and L. L. Yang, "Joint optimization of iterative source and channel decoding using over-complete sourcemapping," to be appeared in Proceedings of IEEE 66th Vehicular Technology Conference VTC2007-Fall, Baltimore, USA, 30 September-3 October 2007.

[2] A. Q. Pham, J. Wang, L. L. Yang, and L. Hanzo, "Joint sourcechannel decoding for an iterative detection aided unequal error protection wavelet video scheme using irregular convolutional codes," IEEE 63rd Vehicular Technology Conference, Melbourne, Australia,, pp. 2484 - 2488, May 2006.

[3] D. Marpe, H. Schwarz, and T. Wiegand, "Context-based adaptive binary arithmetic coding in the h.264/avc video compression standard," IEEE Transactions on Circuits and Systems for Video Technology, vol. Vol. 13, Issue 7, pp. pp. 620-636, July 2003.

[4] F. Kuo and L. Hanzo, "Symbol-flipping based decoding of generalized low-density parity-check codes over GF(q)," Proceedings of IEEE WCNC 2006, vol. 138, pp. 1207-1211, 3-6 April 2006.

[5] S. ten Brink, "Convergence behaviour of iteratively decoded parallel concatenated codes," IEEE Transactions on Communications, pp. 1727-1737, October 2001. 